\title{
Avaliação do desenvolvimento psicomotor pelos médicos de família: estudo observacional
}

Ana Rita Maia, ${ }^{1}$ João Fernandes, ${ }^{2}$ Mariana Fidalgo Leite, ${ }^{3}$ Helena Santos, ${ }^{4}$ Susana Aires Pereira ${ }^{4}$

\section{RESUMO}

Objetivos: Compreender a realidade da avaliação do desenvolvimento psicomotor no âmbito das consultas de vigilância de saúde infantil nas unidades de cuidados de saúde primários dos ACES Gaia e Espinho/Gaia.

Tipo de estudo: Observacional, transversal e descritivo.

Local: ACES Gaia e Espinho/Gaia.

População: Médicos de família dos ACES Gaia e Espinho/Gaia.

Métodos: Aplicação de um questionário de autopreenchimento elaborado pelos autores, com 18 perguntas de resposta múltipla relativas à avaliação do desenvolvimento psicomotor, no âmbito das consultas de saúde infantil. Os dados foram analisados com recurso ao programa Microsoft Excel $2010^{\circledR}$. Este estudo é reportado de acordo com as linhas de orientação STROBE. Resultados: Participaram no estudo 112 dos 193 médicos dos ACES Gaia e Espinho/Gaia. Noventa e nove por cento dos inquiridos avaliam o desenvolvimento psicomotor dos seus utentes e $84 \%$ despendem mais de cinco minutos nessa avaliação; $97 \%$ utilizam a Escala de Mary Sheridan Modificada. Setenta e dois por cento dos inquiridos julgam necessitar de formação sobre a aplicação desta escala; $50 \%$ sentem dúvidas relativamente à referenciação. Setenta e seis por cento consideram a falta de tempo e $66 \%$ a falta de material como sendo as principais dificuldades na avaliação do desenvolvimento.

Conclusões: Tendo em conta o papel fulcral dos médicos de família na avaliação do desenvolvimento psicomotor de lactentes e crianças, torna-se essencial proporcionar a estes profissionais condições de tempo, formação e material adequadas para o cabal desempenho desta tarefa.

Palavras-chave: Desenvolvimento Psicomotor; Cuidados de Saúde Primários.

\section{INTRODUÇÃO}

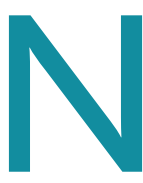

as últimas décadas, o desenvolvimento psicomotor tem merecido um interesse crescente na comunidade pediátrica. A Organização Mundial da Saúde estima que $10 \%$ da população de qualquer país é constituída por pessoas com algum tipo de deficiência. Assim, estima-se que haverá, em Portugal, cerca de um milhão de indivíduos com este tipo de alterações. ${ }^{1}$ Nos Estados Unidos da América, $17 \%$ das crianças e adolescentes apresentam alterações do desenvolvimento em algum momento da sua vida. ${ }^{2-3} \mathrm{O}$ impacto social, emocional e psicológico

1Médica interna de Medicina Geral e Familiar. USF S. Miguel, ACES Espinho/Gaia. ${ }^{2}$ Médico interno de Medicina Geral e Familiar. USF Arco do Prado, ACES Gaia. ${ }^{3}$ Médica interna de Medicina Geral e Familiar. USF Saúde no Futuro, ACES Gaia. ${ }^{4}$ Pediatra. Serviço de Pediatria do Centro Hospitalar de Vila Nova de Gaia/Espinho (Consulta de Desenvolvimento). destas alterações reflete-se não só na criança, como na sua família e na sociedade. O benefício da intervenção precoce é amplamente reconhecido, pelo que o diagnóstico atempado destas situações é essencial. ${ }^{1-2,4-6}$

A Academia Americana de Pediatria recomenda a integração de todas as crianças em programas de vigilância do desenvolvimento psicomotor ${ }^{7}$ que incluam a aplicação de instrumentos de rastreio validados aos nove, dezoito e trinta meses. Também se aconselha a referenciação das crianças em risco de atraso do desenvolvimento para uma consulta especializada, de forma a serem avaliadas e, se necessário, incluídas num programa de intervenção precoce. A Direção-Geral da Saúde publicou, em 2013, o novo Programa Nacional de Saúde Infantil e Juvenil, o qual estabelece a avaliação do desenvolvimento psicomotor como um dos seus grandes objetivos, apresentando um texto de apoio 
exclusivamente dedicado a este tema. ${ }^{8} \mathrm{Na}$ sequência da implementação deste programa, a vigilância em vigor em Portugal assemelha-se à preconizada pela Academia Americana de Pediatria. No entanto, não é proposta a aplicação de testes de rastreio globais nas idades-chave (nove, dezoito e trinta meses), sendo apenas recomendada a utilização do questionário Modified Checklist for Autism in Toddlers, vulgo M-CHAT, entre os 16 e os 30 meses.

Devido à sua natureza sequencial, a avaliação isolada do desenvolvimento psicomotor apresenta valor limitado. Com efeito, a avaliação contínua e longitudinal do desenvolvimento aumenta a sensibilidade e a especificidade para a deteção de alterações. ${ }^{5-6}$ Os médicos de família encontram-se numa posição privilegiada para monitorizar o desenvolvimento psicomotor, uma vez que acompanham as crianças ao longo do tempo e estão, geralmente, familiarizados com a sua história pessoal, social e familiar, assim como com os recursos

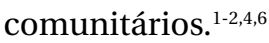

Um estudo conduzido por Figueiras e colaboradores, ${ }^{1}$ que pretendia avaliar os conhecimentos e práticas relacionados com a vigilância do desenvolvimento psicomotor em profissionais dos cuidados de saúde primários, no Brasil, constatou que cerca de $30 \%$ dos profissionais não realizavam esta avaliação por rotina e que, dos restantes, mais de $60 \%$ executavam uma avaliação clínica informal, baseada no senso clínico. No entanto, esta abordagem, quando utilizada de forma isolada, deteta menos de $30 \%$ das crianças com alterações do desenvolvimento. Acresce que menos de metade das alterações importantes é diagnosticada antes dos cinco anos. ${ }^{4,8}$ Em contraste, os instrumentos de rastreio padronizados apresentam sensibilidade e especificidade de 70 a $90 \%{ }^{8}$

As principais dificuldades sentidas nos cuidados de saúde primários, relativamente à vigilância do desenvolvimento psicomotor, são: tempo de consulta reduzido, indisponibilidade de material, formação insuficiente, experiência reduzida na utilização dos testes de rastreio e desconhecimento dos critérios de referenciação. ${ }^{1,4,9}$

Em 2010, Barbeiro e Temudo conduziram um estudo que revelou que mais de metade dos médicos de família do Agrupamento de Centros de Saúde (ACES) Porto Ocidental referia ter dificuldade em interpretar os resultados da Escala de Mary Sheridan Modificada, uma escala de apoio à avaliação do desenvolvimento psicomotor. Os médicos inquiridos consideravam ainda que este teste apresentava numerosas limitações, entre as quais a ausência de um score de referenciação. ${ }^{9}$

Com este estudo pretende-se compreender a realidade da avaliação do desenvolvimento psicomotor no âmbito das consultas de vigilância de saúde infantil nas unidades de Cuidados de Saúde Primários dos ACES Gaia e Espinho/Gaia.

\section{MÉTODOS}

Conduzimos um estudo observacional, transversal e descritivo, abrangendo os médicos especialistas em medicina geral e familiar dos ACES Gaia e Espinho/ /Gaia.

Criou-se um questionário a partir daquele concebido por Barbeiro e Temudo. ${ }^{9}$ O questionário (Apêndice A), de resposta anónima e autopreenchimento, é composto por 18 perguntas de escolha múltipla - as perguntas 1, 6, 7, 10 e 17 permitem a seleção de uma ou mais opções; as perguntas 1, 6, 10 e 17 admitem respostas em texto livre quando é selecionada a opção «Outros»; as perguntas restantes requerem a escolha de uma única opção. As perguntas 1 e 2 incidem sobre a realização da avaliação do desenvolvimento psicomotor; as perguntas 3 a 8 reportam-se às escalas utilizadas nessa avaliação; as perguntas 9 a 11 referem-se ao material utilizado na avaliação do desenvolvimento psicomotor; as perguntas $12 \mathrm{e} 18$ aludem às orientações contidas no Programa Nacional de Saúde Infantil e Juvenil; as perguntas 14 e 15 incidem sobre a referenciação aos cuidados de saúde secundários; as perguntas 16 e 17 reportam-se às necessidades e barreiras à avaliação do desenvolvimento psicomotor.

O questionário contém quatro perguntas diretamente relacionadas com a Escala de Mary Sheridan Modificada (perguntas 3, 4, 8 e 16). Esta escala incide sobre as seguintes componentes do desenvolvimento psicomotor: postura, motricidade global e fina, visão, audição, linguagem, comportamento e adaptação social. É composta por itens a ser avaliados nas idades seguintes: 4-6 semanas; 3, 6, 9, 12 e 18 meses; e 2, 3, 4 e 5 anos. A Escala de Mary Sheridan Modificada está disponível no programa informático de apoio às consultas médicas em uso nos ACES Gaia e Espinho/Gaia, o SAM/SClínico®. 
Foram elaboradas duas versões do questionário (em papel e eletrónica); cada participante escolheu a respetiva modalidade de resposta. A distribuição e a recolha dos questionários decorreram entre maio e setembro de 2014. Foram contactadas todas as unidades de saúde familiar (USF) e unidades de cuidados de saúde personalizados (UCSP) dos ACES Gaia e Espinho/Gaia e incluidos todos os médicos e centros interessados em participar. Excluíram-se os médicos que não exercessem como médicos de família nos ACES Gaia e Espinho/Gaia e os médicos internos.

Os dados recolhidos foram submetidos a uma análise descritiva, com recurso ao programa Microsoft Excel $2010{ }^{\circledR}$. Foram utilizadas as linhas de orientação Strengthening the reporting of observational studies in epidemiology, vulgo STROBE, para a elaboração do presente artigo. ${ }^{10}$

A realização deste estudo foi aprovada pelos Presidentes dos Conselhos Clínicos e de Saúde dos ACES Gaia e Espinho/Gaia e pela Unidade de Investigação Clínica da Administração Regional de Saúde do Norte (ARS Norte), tendo merecido parecer favorável por parte da Comissão de Ética da ARS Norte. Todos os participantes deram o seu consentimento de forma informada.

\section{RESULTADOS}

Dos 193 médicos que exercem como médicos de família nos ACES Gaia e Espinho/Gaia, 112 (58\%) aceitaram participar no estudo após fornecerem o seu consentimento informado. Os 112 participantes trabalham em 31 das 38 unidades funcionais de tipologia USF e UCSP dos ACES Gaia e Espinho/Gaia. Não foram recolhidos dados demográficos, por forma a garantir o anonimato dos participantes.

Cento e onze participantes executam a avaliação do desenvolvimento psicomotor das crianças das suas listas de utentes; na maioria dos casos, esta avaliação é feita pelo médico de família, com ou sem o apoio do enfermeiro (Quadro 1). Esta questão permitia assinalar mais do que uma opção. Um dos participantes não respondeu a esta pergunta. $\mathrm{O}$ inquirido que assinalou a resposta «Outro profissional» não especificou qual.

O tempo que os inquiridos despendem na avaliação do desenvolvimento psicomotor consta do Quadro 1. Dois participantes não responderam a esta questão.

\begin{tabular}{|l|c|}
\hline $\begin{array}{l}\text { QUADRO I. Quem avalia o desenvolvimento } \\
\text { psicomotor e quanto tempo despende nessa avaliação }\end{array}$ \\
\hline Profissional que avalia & $\mathbf{N}(\%)$ \\
\hline Médico de Família & $111(99)$ \\
Enfermeiro & $26(29)$ \\
Pediatra comunitário & $1(1)$ \\
Outro profissional & $1(1)$ \\
Psicólogo & 0 \\
\hline Tempo despendido & $\mathbf{N}(\%)$ \\
\hline Menos de 5 minutos & $16(14)$ \\
Entre 5 e 10 minutos & $65(58)$ \\
Mais de 10 minutos & $29(26)$ \\
\hline
\end{tabular}

QUADRO II. Utilização da Escala de Mary Sheridan Modificada

Escala de Mary Sheridan Modificada

\begin{tabular}{l|c}
\hline Utilização & $\mathbf{N}(\%)$ \\
\hline Sim & $109(97)$ \\
Não & $3(2)$ \\
\hline Formação & $\mathbf{N}(\%)$ \\
\hline Sim & $57(51)$ \\
Não & $55(49)$ \\
\hline Aplicação & $\mathbf{N}(\%)$ \\
\hline Inquérito aos pais & $5(4)$ \\
Observação direta & $4(4)$ \\
As duas opções anteriores & $102(91)$ \\
\hline
\end{tabular}

O Quadro 2 reúne as respostas às questões relativas à utilização da Escala de Mary Sheridan Modificada. Verifica-se que 109 inquiridos (97\%) utilizam esta escala e que 57 (51\%) referem ter recebido formação sobre a aplicação da mesma. Cento e dois participantes (91\%) aplicam a Escala através de inquérito aos pais e observação direta da criança. Um dos participantes não respondeu qual o método que utiliza para aplicar a Escala.

Relativamente ao material utilizado (Quadro 3), 61 inquiridos (54\%) utilizam o material recomendado; dois participantes não responderam a esta questão. Sete inquiridos (6\%) referem que o material é disponibilizado 


\begin{tabular}{|c|c|}
\hline \multicolumn{2}{|c|}{$\begin{array}{l}\text { QUADRO III. Material utilizado para a aplicação da } \\
\text { Escala de Mary Sheridan Modificada }\end{array}$} \\
\hline Material & $\mathbf{N}(\%)$ \\
\hline Usa & $61(54)$ \\
\hline Não usa & $49(44)$ \\
\hline Disponibilizado na USF & $7(6)$ \\
\hline Parcialmente disponibilizado na USF & $23(21)$ \\
\hline Não disponibilizado na USF & $82(73)$ \\
\hline Livro com figuras & $65(58)$ \\
\hline Roca & $50(45)$ \\
\hline Cubos $(2,5 \mathrm{~cm} \times 2,5 \mathrm{~cm})$ & 49 (44) \\
\hline Bola pequena & $47(42)$ \\
\hline Painel com cores e cartões de correspondência & $47(42)$ \\
\hline Bola pendente & $46(41)$ \\
\hline Boneca & $43(38)$ \\
\hline Colher, garfo e escova de cabelo & $24(21)$ \\
\hline Copo ou caneca com asa & $23(21)$ \\
\hline Pinos ou clip & $17(15)$ \\
\hline Outro & $5(4)$ \\
\hline
\end{tabular}

na totalidade pelas respetivas unidades de saúde, 23 participantes $(21 \%)$ referem que dispõem do mesmo apenas parcialmente e 82 médicos $(73 \%)$ responderam que o material não está disponível nas suas unidades de saúde.

A pergunta 10 do questionário refere-se ao material utilizado aquando da aplicação da Escala de Mary Sheridan Modificada; esta questão admitia várias opções de resposta (Quadro 3).

Sete participantes $(6 \%)$ referem utilizar outras escalas para a avaliação do desenvolvimento psicomotor (Quadro 4). Os dois participantes que assinalaram a opção «Outra(s)», não especificaram quais. Relativamente às motivações para a utilização de outras escalas, o questionário permitia a escolha de várias opções (Quadro 4). Os inquiridos que selecionaram a opção «Outro(s)» não preencheram o campo de texto livre adjacente.

Relativamente às orientações contidas no
Programa Nacional de Saúde Infantil e Juvenil, no que concerne à avaliação do desenvolvimento psicomotor 76 participantes (68\%) consideram-nas esclarecedoras, 24 (21\%) discordam desta perceção e 12 (11\%) referem não estar familiarizados com estas orientações.

Cinquenta e seis inquiridos (50\%) referem ter dúvidas acerca do que valorizar e/ou sobre quando referenciar. O Quadro 5 enumera os critérios utilizados pelos participantes para a referenciação de situações suspeitas para os cuidados de saúde secundários. Esta questão admitia a seleção de mais do que uma alínea.

A utilização de um score quantitativo poderia facilitar a referenciação, na opinião de 97 inquiridos (87\%). Oitenta e um participantes (72\%) consideram necessitar de formação para uma melhor aplicação da Escala de Mary Sheridan Modificada.

As principais dificuldades sentidas pelos inquiridos na avaliação do desenvolvimento psicomotor constam do Quadro 6. Esta questão admitia a seleção de mais do que uma opção. O participante que selecionou a opção «Outra(s)» não especificou qual(ais) a(s) dificuldade(s) a que se referia.

Dos 112 inquiridos, 86 (77\%) conversam com os pais ou cuidadores acerca das atividades promotoras do desenvolvimento, 20 (18\%) apenas o faz ocasionalmente e $6(5 \%)$ não tem por hábito fornecer estas informações.
QUADRO IV. Material utilizado para a aplicação da Escala de Mary Sheridan Modificada

Outras escalas de avaliação do desenvolvimento psicomotor

\begin{tabular}{l|l}
\hline Schedule of Growing Skills Ilb & $4(4)$ \\
Teste de Denver & $2(2)$ \\
Teste de Griffiths & 0 \\
Outra(s) & $2(2)$ \\
\hline Motivos & $\mathbf{N}(\%)$ \\
\hline Facilidade de aplicação & $7(6)$ \\
Informação bibliográfica & $6(5)$ \\
Recomendação do local de trabalho e/ou acordo entre os & $2(2)$ \\
colegas do local de trabalho & \\
Recomendação de médico pediatra & 0 \\
Outro(s) & $2(2)$
\end{tabular}




$\begin{aligned} & \text { QUADRO V. Critérios de referenciação para uma } \\
& \text { consulta especializada }\end{aligned}$
\begin{tabular}{l|c} 
Critérios & N (\%) \\
\hline $\begin{array}{l}\text { Sinais de alarme e perceção clínica } \\
\text { Alteração de } 2 \text { ou } 3 \text { itens na Escala de Sheridan } \\
\text { ou presença de } 2 \text { ou } 3 \text { sinais de alarme }\end{array}$ & $12(11)$ \\
$\begin{array}{l}\text { Alteração de } 1 \text { item na Escala de Sheridan ou } \\
\text { presença de } 1 \text { sinal de alarme }\end{array}$ & $11(10)$ \\
$\begin{array}{l}\text { Alteração de mais de } 3 \text { itens na Escala de Sheridan } \\
\text { ou presença de mais de } 3 \text { sinais de alarme }\end{array}$ & $2(2)$
\end{tabular}

\begin{tabular}{l|l}
$\begin{array}{l}\text { QUADRO VI. Dificuldades sentidas na avaliação do } \\
\text { desenvolvimento psicomotor }\end{array}$ \\
\begin{tabular}{l|c} 
Dificuldades & N (\%) \\
\hline Falta de tempo & $85(76)$ \\
$\begin{array}{l}\text { Falta de material } \\
\text { Formação insuficiente sobre a aplicação das } \\
\text { escalas de avaliação }\end{array}$ & $74(66)$ \\
$\begin{array}{l}\text { Falta de prática na aplicação das escalas de } \\
\text { avaliação }\end{array}$ & $41(37)$ \\
Outra(s) & $21(19)$ \\
\end{tabular}
\end{tabular}

\section{DISCUSSÃO}

Verifica-se que a avaliação do desenvolvimento psicomotor dos lactentes e crianças, aquando das consultas de saúde infantil, é um procedimento rotineiro e generalizado entre os médicos de família inquiridos.

Destaca-se que os médicos são quem mais realiza esta avaliação, embora o enfermeiro também tenha um papel ativo nesta avaliação em cerca de um terço dos casos. A pediatria comunitária, disponível apenas em algumas das unidades avaliadas neste estudo, parece ter pouca expressão nos ACES Gaia e Espinho/Gaia.

Relativamente ao tempo dedicado a esta avaliação, sublinha-se que mais de metade dos inquiridos despendem entre cinco e dez minutos e cerca de um terço despende mais de dez minutos, o que representa cerca de um quarto a metade do tempo de duração estabelecido para as consultas de saúde infantil e juvenil.

A Escala de Mary Sheridan Modificada disponível no programa SAM/SClínico ${ }^{\circledR}$ é utilizada pela quase tota- lidade dos inquiridos. Este facto poderá dever-se, em parte, por ser esta a escala recomendada para a avaliação do desenvolvimento psicomotor no Programa Nacional de Saúde Infantil e Juvenil e por esta se encontrar disponível e facilmente acessível no programa de saúde infantil do SAM/SClínico®.

No que à aplicação da Escala de Mary Sheridan Modificada diz respeito, a maioria dos inquiridos respondeu que utilizava quer o inquérito aos pais quer a observação direta da criança. Destaque-se que cinco participantes apenas inquirem os pais. Tal facto pode levar a interpretações erradas acerca das capacidades da criança ainda que, como é referido no Programa Nacional de Saúde Infantil e Juvenil, "os pais, familiares e educadores acompanham a criança durante muito mais tempo do que os profissionais de saúde e convivem com ela num meio onde a mesma se expressa mais à vontade". ${ }^{8}$ Assim, é importante que, quando questionados, os pais/educadores compreendam o que se pretende saber que a criança é capaz de fazer. Os pais/educadores são uma fonte preciosa de informação que não deve ser menosprezada. ${ }^{5}$

Relativamente ao material necessário para a aplicação da Escala, este não é disponibilizado pela maioria das unidades (73\% dos inquiridos). Metade (49\%) dos participantes não recebeu qualquer formação sobre a aplicação desta escala; $72 \%$ referem necessitar dessa formação. Pode conduzir, por um lado, à aplicação incorreta da Escala e, consequentemente, à obtenção de resultados enviesados, ou à aplicação apenas parcial da mesma. Ao encontro desta ideia vão as respostas sobre as dificuldades sentidas na avaliação do desenvolvimento psicomotor: a falta de tempo e de material.

No que concerne às orientações do Programa Nacional de Saúde Infantil e Juvenil relativamente à avaliação do desenvolvimento psicomotor, a maioria dos inquiridos considera-as esclarecedoras; $11 \%$ admitem não estar familiarizados com este documento. De notar que o questionário elaborado foi respondido um ano após a introdução do Programa Nacional de Saúde Infantil e Juvenil. O Programa-tipo anterior não incluía orientações tão detalhadas relativamente à avaliação do desenvolvimento psicomotor.

Destaca-se positivamente que $77 \%$ dos médicos conversa com os pais ou cuidadores sobre as atividades promotoras do desenvolvimento referidas no Programa Nacional de Saúde Infantil e Juvenil. 
Por último, relativamente à referenciação à consulta de pediatria nos cuidados de saúde secundários, metade dos inquiridos refere ter dúvidas acerca do momento adequado; esta decisão baseia-se, na maioria dos casos, em sinais de alarme e perceção clínica. Apenas $23 \%$ dos participantes assinalam ter por base as alterações na Escala de Mary Sheridan Modificada e/ou os sinais de alarme (também disponíveis no programa SAM/SClínico®). Pode discutir-se se os médicos de família continuam a valorizar mais o senso clínico e, mesmo aplicando a Escala de Mary Sheridan Modificada, não a valorizam da mesma forma. Ou se, por outro lado, não interpretam as alterações na Escala de Mary Sheridan Modificada como critérios estanques de referenciação, dado que podem reavaliar a criança de forma sequencial e num curto espaço de tempo (uma das prerrogativas da medicina geral e familiar). Ainda assim, o facto de $50 \%$ dos inquiridos referirem dúvidas acerca dos critérios de referenciação e considerarem úteis scores quantitativos para apoio à decisão parece merecedor de reflexão posterior.

Importa destacar que este estudo permitiu conhecer a realidade da avaliação do desenvolvimento psicomotor nos ACES Gaia e Espinho/Gaia e os resultados obtidos apontam as mesmas limitações encontradas, em 2010, por Barbeiro e Temudo no ACES Porto Ocidental: ${ }^{9}$ os inquiridos que utilizavam a Escala de Mary Sheridan Modificada referiam dificuldades em interpretar os resultados, mais de um terço não considerava claras as orientações da Direção-Geral da Saúde sobre a avaliação do desenvolvimento psicomotor e somente $21 \%$ dos médicos tinha realizado formação sobre a utilização da Escala de Mary Sheridan Modificada. Nesse estudo, como neste, os médicos de família inquiridos apontavam que a ausência de um score de referenciação era também uma das principais limitações desta Escala. Verifica-se, em ambos os estudos, que os critérios de referenciação das crianças em risco não são uniformes, o que reflete a não existência de diretivas ou o desconhecimento das mesmas.

Como limitações do presente estudo destaca-se o envolvimento de um número restrito de médicos de família com a obtenção de uma amostra não representativa da realidade nacional. A ausência de recolha de dados sobre os médicos não respondedores impede a caracterização desta parte da população estudada. O lo- cal de trabalho dos médicos inquiridos (USF vs UCSP) poderá também ter influenciado os resultados obtidos, uma vez que o acesso a recursos (nomeadamente materiais) e a contratualização de indicadores no âmbito da saúde infantil pode levar a um maior investimento na avaliação do desenvolvimento psicomotor. A utilização de um questionário desenvolvido pelos autores poderá ter conduzido a viéses de informação; o facto de o questionário aplicado conter maioritariamente questões de resposta fechada poderá ter contribuído para este viés. Acresce que alguns dos participantes poderão ter sentido a necessidade de optar pelas respostas que considerassem mais corretas, ao invés das respostas mais representativas da sua prática clínica. Por fim, a obtenção de respostas em branco ou incompletas dificulta a interpretação dos dados obtidos.

Assim, considerou-se ser necessário melhorar a divulgação do Programa Nacional de Saúde Infantil e Juvenil e promover sessões dirigidas a médicos de família sobre a avaliação do desenvolvimento psicomotor, assim como garantir a disponibilidade do material necessário à sua avaliação nas unidades de saúde. Num cenário ideal, a duração das consultas de saúde infantil deveria ser alargada.

Por outro lado, as Unidades Coordenadoras Funcionais desempenham um papel fundamental na articulação entre a medicina geral e familiar e as especialidades hospitalares. O estabelecimento de critérios de referenciação, assim como a eventual criação de um score de referenciação quantitativo ao nível destas unidades poderia facilitar este processo. Com efeito, a Escala de Mary Sheridan Modificada, concebida para apoiar as avaliações seriadas do desenvolvimento psicomotor, não constitui um instrumento de rastreio de alterações do desenvolvimento psicomotor. Assim, poderá ser útil a implementação de consultas de rastreio, a realizar em idades-chave, para aplicação de escalas validadas por parte de profissionais de saúde treinados para o efeito.

\section{REFERÊNCIAS BIBLIOGRÁFICAS}

1. Figueiras AC, Puccini RF, Silva EM, Pedromonico MR. Avaliação das práticas e conhecimentos de profissionais da atenção primária à saúde sobre vigilância do desenvolvimento infantil [Evaluation of practices and knowledge among primary health care professionals in relation to child development surveillance]. Cad Saúde Pública. 2003;19(6):1691-9. Portuguese 
2. Allen SG, Berry AD, Brewster JA, Chalasani RK, Mack PK. Enhancing developmentally oriented primary care: an Illinois initiative to increase developmental screening in medical homes. Pediatrics. 2010;126 Suppl 3:5160-4.

3. Jensen RE, Chan KS, Weiner JP, Fowles JB, Neale SM. Implementing electronic health record-based quality measures for developmental screening. Pediatrics. 2009;124(4):e648-54.

4. Aly Z, Taj F, Ibrahim S. Missed opportunities in surveillance and screening systems to detect developmental delay: a developing country perspective. Brain Dev. 2010;32(2):90-7.

5. Pinto M. Vigilância do desenvolvimento psicomotor e sinais de alarme [The surveillance and warning signs of child's development]. Rev Port Clin Geral. 2009;25(6):677-87. Portuguese

6. Oliveira G. Autismo: diagnóstico e orientação. Parte I - Vigilância, rastreio e orientação nos cuidados primários de saúde [Autism: diagnosis and management. Part I - Monitoring, screening and management in primary health care]. Acta Pediatr Port. 2009;40(6):278-87. Portuguese

7. Council on Children with Disabilities, Section on Developmental Behavioral Pediatrics, Bright Futures Steering Committee, Medical Home Initiatives for Children with Special Needs Project Advisory Committee. Identifying infants and young children with developmental disorders in the medical home: an algorithm for developmental surveillance and screening. Pediatrics. 2006;118(1):405-20.

8. Direção-Geral da Saúde. Programa nacional de saúde infantil e juvenil: norma da DGS nº 010/2013, de 31/05/2013. Lisboa: DGS; 2013.
9. Barbeiro S. Avaliação das etapas do desenvolvimento psicomotor dos 0 aos 24 meses segundo o teste de Haizea-Llevant: demonstração por vídeo [Dissertation]. Porto: Instituto de Ciências Biomédicas Abel Salazar, Universidade do Porto; 2011. Available from: http://repositorioaberto.up.pt/bitstream/10216/53704/2/Tese\%20\%20Sandra\%20Barbeiro.pdf

10. von Elm E, Altman DG, Egger M, Pocock SJ, Gøtzsche PC, Vandenbroucke $J$, et al. The Strengthening the Reporting of Observational Studies in Epidemiology (STROBE) statement: guidelines for reporting observational studies. J Clin Epidemiol. 2008r;61(4):344-9.

\section{CONFLITO DE INTERESSES}

Os autores declaram não ter conflitos de interesses.

\section{COMISSÃO DE ÉTICA}

Estudo realizado após parecer favorável da Comissão de Ética da ARS Norte.

\section{ENDEREÇO PARA CORRESPONDÊNCIA \\ Ana Rita Maia \\ Av. da Igreja, n. ${ }^{\circ}$ 311, 4410-411 Arcozelo, VNG \\ E-mail: rita.rmaia@gmail.com}

Recebido em 30-03-2015

Aceite para publicação em 25-11-2015

\section{ABSTRACT}

\section{EVALUATION OF PSYCHOMOTOR DEVELOPMENT IN CHILDREN BY FAMILY DOCTORS: AN OBSERVATIONAL STUDY}

Goals: To assess the current status of the evaluation of psychomotor development in children by family doctors during regular clinic visits in two primary healthcare groupings, ACES Gaia and Espinho/Gaia.

Study design: Cross-sectional study.

Location: ACES Gaia and Espinho/Gaia.

Population: Family medicine specialists of ACES Gaia and Espinho/Gaia.

Methods: We distributed a self-administered questionnaire written by the authors consisting of 18 multiple-choice questions about the evaluation of psychomotor development during well child consultations. Data were analyzed with Microsoft Excel $2010^{\circledR}$. This study is reported according to the STROBE guidelines.

Results: Completed questionnaires were received from 112 of the 193 specialists (58\%) in these two healthcare groupings. The assessment of psychomotor development is performed by $99 \%$ and $84 \%$ take more than five minutes in doing so. The Modified Mary Sheridan Scale is used by $97 \%$ and $72 \%$ of respondents considered that they need more training on the use of this scale, while $50 \%$ have doubts related to referrals. A lack of time ( $76 \%$ of respondents) and a lack of material ( $66 \%$ of respondents) are considered to be the main difficulties encountered when assessing psychomotor development.

Conclusions: Given the role that family doctors play in the assessment of the psychomotor development of infants and children, it is essential to provide these professionals with adequate time, training, and material conditions to complete this task.

Keywords: Psychomotor Development; Primary Healthcare. 


\section{APÊNDICE A}

Questionário aplicado

\section{Avaliação do desenvolvimento psicomotor - utilização da Escala de Mary Sheridan \\ Modificada nos Cuidados de Saúde Primários}

\section{QUESTIONÁRIO}

1. Quem realiza a avaliação do desenvolvimento psicomotor às crianças da sua lista de utentes?

Pode escolher mais do que uma opção.
a. Médico de família
b. Pediatra comunitário
c. Enfermeiro
d. Psicólogo
e. Outro profissional

f. O desenvolvimento psicomotor dos meus utentes não é avaliado

2. Quanto tempo despende nesta avaliação?

$\square$ a. $<5 \mathrm{~min}$.

b. $5-10 \mathrm{~min}$.

c. $>10 \mathrm{~min}$.

3. Utiliza a Escala de Mary Sheridan Modificada disponível no programa SAM?
a. Sim
b. Não

4. Recebeu formação sobre a aplicação da Escala de Mary Sheridan Modificada?
a. $\operatorname{Sim}$
b. Não

5. Utiliza outra(s) escala(s)?

a. Sim

b. Não

Caso tenha respondido "Não" à pergunta anterior, queira passar para a pergunta $n .^{\circ} 8$.

6. Qual(ais)?
c. Shedule of Growing Skills II
d. Teste de Denver
e. Teste de Griffiths
f. Outra(s)

7. Por que motivo optou por usar essa(s) escala(s)?
a. Recomendação de médico pediatra
b. Recomendação do local de trabalho e/ou acordo entre os colegas do local de trabalho
c. Facilidade de aplicação
d. Informação bibliográfica
e. Outro(s)

8. Como aplica a Escala de Mary Sheridan Modificada?
a. Inquérito aos pais
b. Observação direta
c. As duas opções anteriores

9. Utiliza o material aconselhado para a aplicação da escala?
a. Sim
b. Não

10. Qual o material que utiliza? Pode escolher mais do que uma opção.
a. Bola pendente
b. Roca
c. Cubos $(2,5 \mathrm{~cm} \times 2,5 \mathrm{~cm})$
d. Pinos ou clip
e. Boneca
f. Colher, garfo e escova de cabelo
g. Livro com figuras
h. Copo ou caneca com asa
i. Bola pequena
j. Painel com cores
k. Outro

11. O material é disponibilizado pela sua unidade de saúde?
a. Sim
b. Parte do material
c. Não 
12. Considera esclarecedoras as orientações contidas no Programa Nacional de Saúde Infantil e Juvenil no que concerne a avaliação do desenvolvimento psicomotor?
a. Sim
b. Não
c. Não estou familiarizado(a) com as orientações em apreço

13. Tem dúvidas sobre o que valorizar e/ou sobre quando referenciar?
a. Sim
b. Não

14. Que critérios utiliza para referenciar? Nota: MSM - (Escala de) Mary Sheridan Modificada

a. Sinais de alarme e perceção clínica

b. Alteração de 1 item na escala de MSM ou presença de 1 sinal de alarme

c. Alteração de 2 ou 3 itens na escala de MSM ou presença de 2 ou 3 sinais de alarme

d. Alteração de mais de 3 itens na escala de MSM ou presença de mais de 3 sinais de alarme

15. Considera que a utilização de um score quantitativo poderia facilitar a referenciação?
a. Sim
b. Não

16. Considera necessitar de formação para uma melhor aplicação da Escala de Mary Sheridan Modificada?
a. Sim

b. Não

17. Qual(ais) a(s) principal(ais) dificuldade(s) que sente na avaliação do desenvolvimento psicomotor dos seu utentes?

Pode escolher mais do que uma opção.
a. Falta de tempo
b. Falta de material
c. Formação insuficiente sobre a aplicação das escalas de avaliação
d. Falta de prática na aplicação das escalas de avaliação

e. Outra(s)

18. Conversa com os pais ou cuidadores acerca das atividades promotoras do desenvolvimento psicomotor mencionadas no Programa Nacional de Saúde Infantil e Juvenil?
a. Sim
b. Ocasionalmente
c. Não

\section{Dados pessoais}

Qual a unidade de saúde na qual trabalha? 